\title{
Shuttle Debris Impact Tool Assessment Using the Modern Design of Experiments
}

Computational tools have been developed to estimate thermal and mechanical reentry loads experienced by the Space Shuttle Orbiter as the result of cavities in the Thermal Protection System (TPS). Such cavities can be caused by impact from ice or insulating foam debris shed from the External Tank (ET) on liftoff. The reentry loads depend on cavity geometry and certain Shuttle state variables, among other factors.

Certain simplifying assumptions have been made in the tool development about the cavity geometry variables. For example, the cavities are all modeled as "shoeboxes", with rectangular cross-sections and planar walls. So an actual cavity is typically approximated with an idealized cavity described in terms of its length, width, and depth, as well as its entry angle, exit angle, and side angles (assumed to be the same for both sides).

As part of a comprehensive assessment of the uncertainty in reentry loads estimated by the debris impact assessment tools, an effort has been initiated to quantify the component of the uncertainty that is due to imperfect geometry specifications for the debris impact cavities. The approach is to compute predicted loads for a set of geometry factor combinations sufficient to develop polynomial approximations to the complex, nonparametric underlying computational models. Such polynomial models are continuous and feature estimable, continuous derivatives, conditions that facilitate the propagation of independent variable errors.

As an additional benefit, once the polynomial models have been developed, they require fewer computational resources to execute than the underlying finite element and computational fluid dynamics codes, and can generate reentry loads estimates in significantly less time. This provides a practical screening capability, in which a large number of debris impact cavities can be quickly classified either as harmless, or subject to additional analysis with the more comprehensive underlying computational tools. The polynomial models also provide useful insights into the sensitivity of reentry loads to various cavity geometry variables, and reveal complex interactions among those variables that indicate how the sensitivity of one variable depends on the level of one or more other variables. For example, the effect of cavity length on certain reentry loads depends on the depth of the cavity. Such interactions are clearly displayed in the polynomial response models.

Five independent cavity geometry variables were selected to define the polynomial response models, with the decision to hold exit angle fixed at $75^{\circ}$. This decision was predicated on preliminary results that revealed relatively little impact of exit angle changes on computed reentry loads, at least over the range of $70^{\circ}$ to $80^{\circ}$, which prior experience suggests is typical for exit angles. In addition to the five independent geometry variables, two Shuttle state variables were identified. The first, boundary layer transition time, serves as a surrogate for surface roughness and was defined as a categorical variable with two fixed levels, 900 seconds and 1200 seconds, selected to bracket the anticipated range 
of this variable. The second, baseline heating, was likewise established as a two-level categorical variable, representing low and moderate baseline heating conditions.

Even with only five independent numerical variables and two categorical variables, the number of computational points required to develop a third-order polynomial model could be considerable. For example, at least four levels are required in each numerical variable to represent a third-order factor dependence, so a full factorial experiment design involving four levels in five factors would require $4^{5}=1024$ points at each of the four combinations of high and low levels of the two categorical variables, or 4096 points per computational experiment. The intent was to develop such models for each of the 33 Shuttle body point zones, so over 135,000 points would be required by such a comprehensive design. Even at an optimistic computational rate of 10 minutes per point, this would require over 22,000 hours of computer time, a totally unrealistic requirement. For this reason a decision was taken to apply Modern Design of Experiments (MDOE) methods to the development of the 7-factor computational experiments required in each body point zone. MDOE methods were originally introduced at NASA Langley Research Center in 1997 to extend conventional "DOE" (design of experiments) methods to relatively complex and specialized requirements in high-precision aerospace research, notably wind tunnel research. Since then, MDOE methods have been applied to a wide range of physical and computational experiments to reduce costs and uncertainty, and to provide unique insights into factor interactions.

This paper describes the development of MDOE computational experiments to generate response surface models for factor uncertainty propagation in each of the Shuttle's 33 body point zones. Special elements of the design are described, including factor constraints that preclude certain physically unrealizable combinations of low entry angle with short cavity length at specified depths, and shallow side angles with narrow cavities. Other special conditions are also described, including methods to cope with missing data in certain body point zones.

The impact of transformations in both dependent and independent variables is discussed, as are certain special techniques to account for the nonlinear nature of factor constraints in this problem. Scaling issues are described, which drove decisions about data volume requirements. The selection of factor combinations to minimize uncertainty in regression coefficients is also described, as are the numerous tests used to validate the response models, including the analysis of confirmation points for each body point zone. Lack of fit errors quantifying how well the MDOE response models reproduce the underlying computational models are documented.

Finally, representative response models are displayed, illustrating how reentry loads are predicted to depend on cavity geometry for various Shuttle state variable combinations, and the uncertainty in these load predictions due to uncertainty in the cavity dimensions and angles will also be presented. The figures below are examples.

Figure 1 indicates the degree of agreement between the MDOE polynomial response 
surface and the more complex underlying code for the full range of cavity dimensions studied in Shuttle body point zone 1450 (length from 2 in. to $10 \mathrm{in}$., width from 1.4" to 4 ", and depth from $0.1^{\prime \prime}$ to $0.6^{\prime \prime}$, entry angle from $10^{\circ}$ to $60^{\circ}$, and sidewall angle from $10^{\circ}$ to $87^{\circ}$ ). The standard deviation in residuals on the left is $1.26^{\circ} \mathrm{F}$, representing $0.5 \%$ of the mean structure temperature for all cavities modeled. The figure on the right compares structure temperature values estimated with the MDOE polynomial model (predicted) to corresponding values computed by the underlying code ("actual").

Figure 2 shows a representative response surface on the left, representing structure temperatures for a 4 in. wide cavity in body point zone 1450 with a $45^{\circ}$ entry angle and $60^{\circ}$ side angles, for nominal baseline heating and a boundary layer transition time of 1200 seconds. On the right is the corresponding distribution of $95 \%$ confidence interval half widths representing uncertainty in structure temperature that is attributable to uncertainty in cavity geometry variables. The assumed uncertainty in estimating cavity dimensions is $\pm 1 / 8^{\text {th }}$ inch $(3 \sigma)$. The assumed uncertainty in estimating wall angles is $\pm 5^{\circ}(2 \sigma)$.

Note that the response surfaces clearly reveal general features of the response as well as some subtle interaction effects. For example, the structure temperature obviously increases with both the length and the depth of the cavity, but cavity length has a greater effect for deeper cavities than more shallow cavities. That is, a given increase in length will cause a greater increase in structure temperature for deep cavities than shallow cavities, which is consistent with intuitive expectations. Likewise, increasing the depth of a cavity causes the structure temperature to increase more for a longer cavity than a shorter one.

From the plot on the right in figure 2, the uncertainty in structure temperature attributable to uncertainty in cavity geometry appears to be only slightly larger for deep cavities than for shallow ones, but the uncertainty is significantly greater for longer cavities than for shorter ones. This suggests that rather more care is needed in estimating cavity sizes and shapes for longer cavities than shorter ones.

\section{Representative Figures}




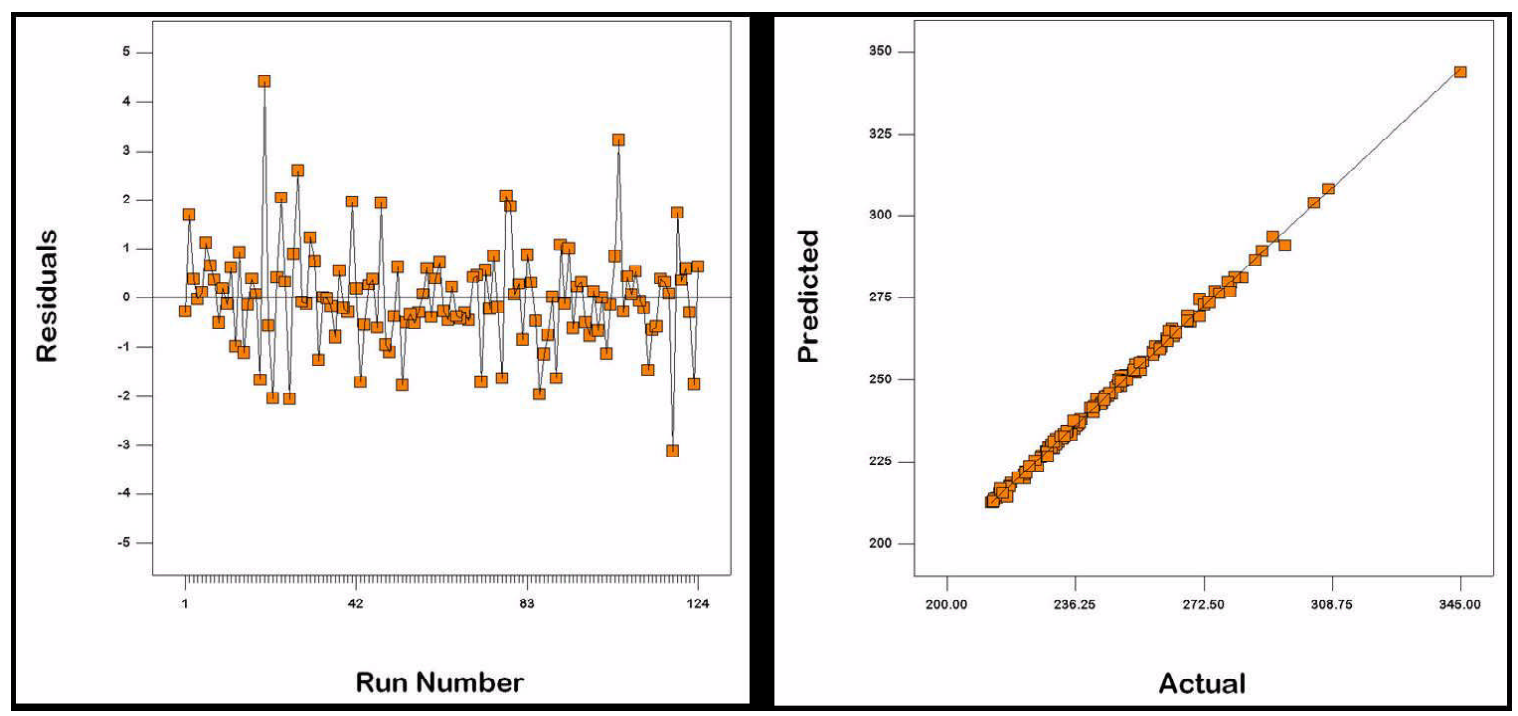

Figure 1: Agreement between MDOE structure temperature model and underlying code for Shuttle body point zone 1450 . Standard deviation in residuals on the left is $1.26^{\circ} \mathrm{F}$.

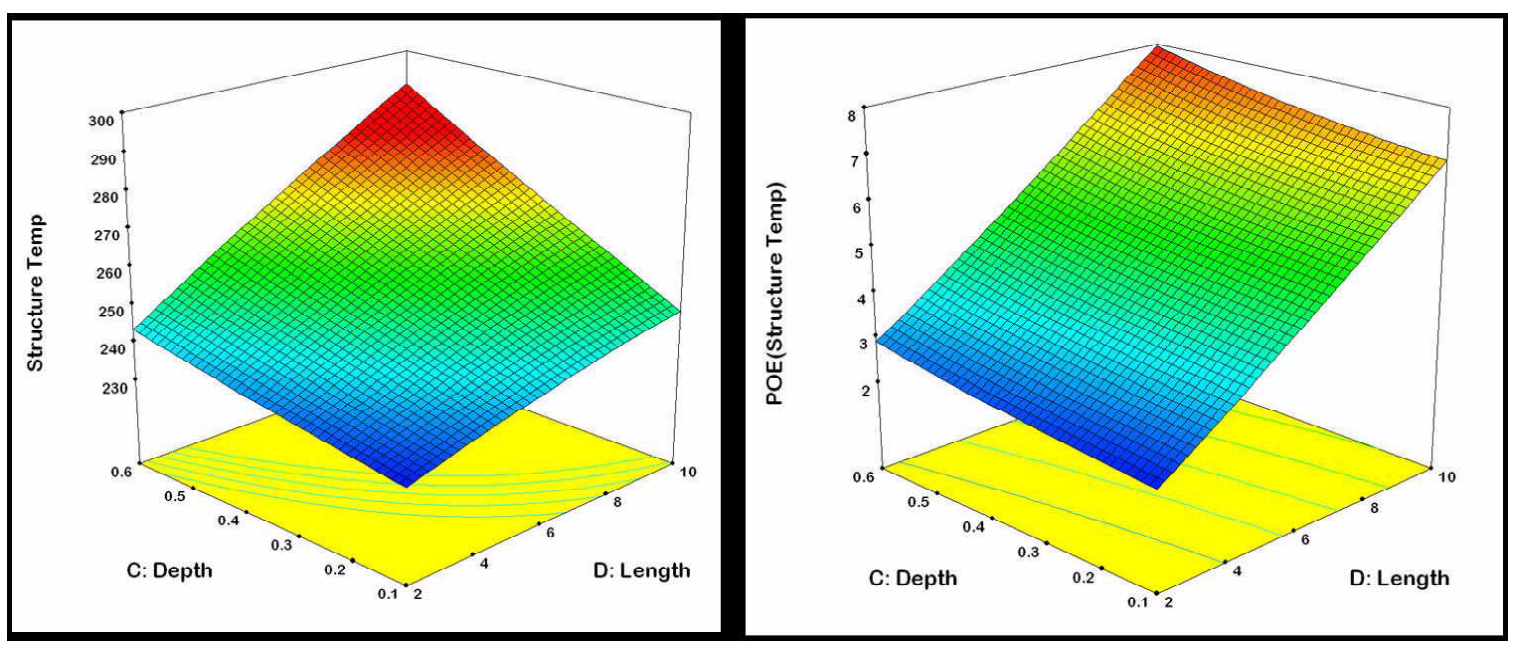

Figure 2, Left: Structure temperature on reentry in Shuttle body point zone 1450 for 4 in. wide cavity with $45^{\circ}$ entry angle and $60^{\circ}$ side angles, for nominal baseline heating and a boundary layer transition time of 1200 seconds. Right: $95 \%$ confidence interval half widths for uncertainty in structure temperature due to cavity geometry uncertainty. 